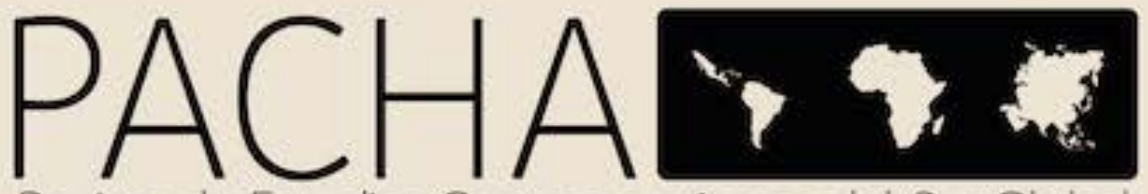

Revista de Estudios Contemporáneos del Sur Global Joumal of Contemporary Studies of the Global South Revista de Estudos Contemporâneos do Sul Global 


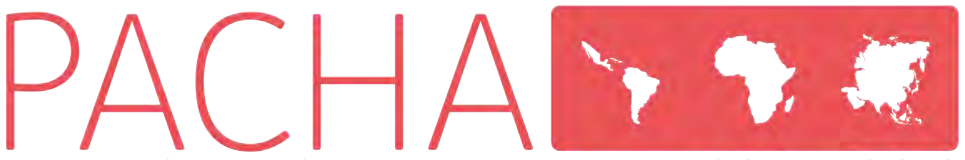

Revista de Estudios Contemporáneos del Sur Global

Joumal of Contemporary Studies of the Global South

\section{Equipo Editorial}

Carolina Díaz R.

Directora Editorial

CICSH-AL Centro de Investigaciones en Ciencias Sociales y Humanidades desde América Latina, Ecuador revistapacha@religacion.com; carolinadiaz@religacion.com

Roberto Simbaña Q.

Coordinador Editorial

CICSH-AL Centro de Investigaciones en Ciencias Sociales y Humanidades desde América Latina, Ecuador robertosimbana@religacion.com

\section{Editores Asociados}

Paola Andrea Tovar. Universidad De Montreal, Colombia. Editora Asociada en Antropología

Mitchell Alberto Alarcón Díaz. Universidad Nacional Mayor de San Marcos, Perú. Editor Asociado en Educación

Marcela Cristina Quinteros. Universidade Estadual de Maringá, Brasil. Editora Asociada en Historia Latinoamericana

Aboutaleb Sedatee Shamir. Islamic Azad University, Iran. Editor Asociado en Educación y Medio Oriente

Mateus Gamba Torres. Universidade de Brasilia - UNB, Brasil. Editor Asociado en Historia Mirna Yazmin Estrella Vega. Universidad Nacional Autónoma de México, México. Editora Asociada en Sociología

Rodrigo Navarrete Saavedra. Universidad Aus tral de Chile, Chile. Editor Asociado en Ciencias Políticas

Aygul Zufarovna Ibatova. Tyumen Industria University, Rusia. Editora Asociada en Humahidades sobre Asia
Shokhrud Fayziev Farmanovich. Tadqiqot, Tashkent, Uzbekistan. Editor Asociado en Desarrollo sobre la Comunidad de Estados Independientes, Asia.

Mª Aránzazu Serantes López. Woolf University, España. Editora Asociada en Humanidades digitales

Fabricio Espinosa Ortiz. Consejo Nacional de Ciencia y Tecnología, México. Editor Asociado en Geografía Humana

Marcelo Starcenbaum. Universidad Nacional de La Plata, Argentina. Editor Asociado en Filosofía e Historiografía

Siti Mistima Maat. Universiti Kebangsaan Malaysia, Malaysia. Editora Asociada en Innovación de aprendizaje

Carla Vanessa Zapata Toapanta. Universidad de Salamanca, España. Editora Asociada en Latinoamericanismo

\section{Consejo Editorial}

Andrea Paola Cantarelli, Universidad Nacional de Cuyo, Argentina
Federico Cabrera, Universidad Nacional de San Juan/CONICET, Argentina

Gamaniel David Suárez Cobix, Universidad Veracruzana, México

Gloria Concepción Tenorio Sepúlveda, Tecnológico de Estudios Superiores de Chalco, México

Héctor García Cornejo, Universidad Michoacana de San Nicolás de Hidalgo, México

Jorge Gilberto Bonilla Macas, Universidad Católica de Cuenca, Ecuador

José Alexander Rubiano Pedroza, Universidad de Pamplona, Colombia

María Dolores Sancho, Universidad Nacional del Comahue, Argentina

Mauricio Sandoval Cordero, Facultad Latinoamericana de Ciencias Sociales, Costa Rica

Rivera Varela Bertha Leticia, Universidad Abierta a Distancia, México

Tomás Sebastián Torres López, Universidad Alberto Hurtado, Chile

\section{CONSEJO ASESOR INTERNACIONAL}

\author{
Alejandro Mejía Tarazona \\ Facultad Latinoamericana de Ciencias Sociales, Ecuador \\ Alexander Luna Nieto \\ Fundación Universitaria de Popayán, Colombia \\ Celeste De Marco \\ CONICET/Universidad Nacional de Quilmes-CEAR, Argentina \\ Christian Andres Quinteros Flores \\ Universidad de Chile, Chile \\ Daniel Orizaga Doguim \\ Centro de Investigaciones Multidisciplinarias-UAQ, México \\ Francisco Javier Jover Mart \\ Universidad de Castilla-La Mancha, España
}

Gaya Makaran

Centro de Investigaciones sobre América Latina y el Caribe CIALC, UNAM, México

Jaime Araujo Frias

Universidad Nacional de San Agustín, Perú

João Luis Binde

Universidade Federal De Pernambuco, Brasil

Luisina Castelli Rodríguez

Universidad de la República, Uruguay

Mariana Jesica Lerchund

Universidad Nacional de Río Cuarto, Argentina

Marina Acosta

Universidad de Buenos Aires, Argentina

Noelia Marina Cortinas

Universidad de Buenos Aires, Argentin

Paulo Alves Pereira Júnior

Universidad Estatal Paulista, Brasil

Sergio Monroy Isaza

Universidad de Ibagué, Colombia

Suyai Malen Garcia Gualda

IPEHCS-CONICET-UNCO, Argentin 


\title{
Acceso, usos y problemas en la educación virtual: una aproximación a las experiencias de estudiantes y docentes durante la cuarentena obligatoria en Argentina*
}

\author{
Access, uses and problems in virtual education: an approach to the experiences of students and teachers during \\ compulsory quarantine in Argentina
}

\section{RESUMEN}

El presente artículo se propone reconstruir un estado de situación a partir de las experiencias y representaciones de docentes y estudiantes, durante el período de cuarentena obligatoria, en el contexto de la pandemia mundial por Covid-19. A partir de ello se intenta contribuir a profundizar el debate acerca de las transformaciones y problemáticas emergentes que están teniendo lugar en el marco de la llamada educación virtual, prestando especial ataención al actual escenario que se abre a la par de la implementación de políticas de confinamiento o aislamiento preventivo. Se espera que una lectura como esta, en clave de acceso, usos y problemas permita seguir de cerca las desigualdades que afectan a los países de nuestra región.

Palabras clave: Plataformas; Educación Virtual; Brecha digital; Cuarentena, Covid-19

\section{ABSTRACT}

This article aims to reconstruct a state of a situation based on the experiences and representations of teachers and students, during the period of compulsory quarantine, in the context of the global pandemic by Covid-19. From this, an attempt is made to contribute to deepening the debate about the transformations and emerging problems that are taking place within the framework of the so-called virtual education, paying special attention to the current scenario that is opening up alongside the implementation of confinement policies or preventive isolation. It is expected that reading like this, in terms of access, uses, and problems will allow us to closely follow the inequalities that affect the countries of our region..

Keywords: Plattforms; Virtual Education; Digital Devide; Quarantine; Covid-19

*El presente artículo forma parte de los resultados del proyecto de Investigación "Trayectorias de Nivel Superior", radicado en la Universidad Nacional de Villa María (Córdoba, Argentina). 


\section{INTRODUCCIÓN}

Aquello que sabemos hoy acerca de la educación virtual no resulta suficiente para comprender la clase de procesos en los que se han visto involucradas las instituciones educativas de la región, luego de la implementación de las políticas de prevención y aislamiento físico que se sucedieron en el marco de la pandemia global del virus Covid-19. En el caso de Argentina, el Decreto de Necesidad y Urgencia $\mathrm{N}^{0} 297$, dispuso la medida de "aislamiento social, preventivo y obligatorio" a partir del 20 de marzo de 2020, lo que incluyó la suspensión del dictado de clases presenciales en todos los niveles de la educación formal. Esto abrió un escenario impensado, en el cual docentes, directivos, estudiantes y padres se vieron obligados a recurrir a los distintos entornos y plataformas virtuales de enseñanza disponibles, pese a no desempeñarse habitualmente, ni mucho menos haber optado por una modalidad de enseñanza virtual o a distancia. De este modo, los actores involucrados se encontraron frente a un verdadero desafío, tanto pedagógico como técnico, en la medida en que algunas de estas herramientas no habían sido pensadas para responder a una demanda con tal masividad. Y esto vale no solo a nivel de software, sino también respecto de la disponibilidad y características de los dispositivos que con que contaban hasta entonces las instituciones y los hogares.

Al llegar a este punto, cabe preguntarnos: ¿estamos frente a una nueva etapa en la virtualización o digitalización de la enseñanza? ¿Es posible que las experiencias recienten hayan contribuido a acelerar algunos de los procesos de digitalización de nuestras vidas? ¿O se trata tan solo de una fase caracterizada por cierta liminalidad, en la cual aún no es posible identificar lo nuevo de lo viejo? Ante la imposibilidad de aportar certezas respecto al devenir del campo educativo, debemos estar dispuestos al menos a reconocer que estas experiencias abren un horizonte de nuevas posibilidades, aunque también de nuevas problemáticas que precisan ser abordadas.

El principal objetivo de este trabajo apunta a construir un mapa de coordenadas acerca de las principales problemáticas emergentes en materia educativa, en contextos de confinamiento o asilamiento preventivo. Se espera que una lectura como esta, en clave de acceso, usos y problemas, permita orientar múltiples lecturas acerca de un estado de situación caracterizado por la urgencia y la transformación constante. A pesar de lo paradójico que pueda resultar esto, no debemos soslayar la necesidad de contar en lo inmediato con un conjunto de datos que permitan dar cuenta de este presente, por más cambiante que nos resulte. Pues esto es lo que permitirá ampliar o reorientar la mirada en el futuro.

Al indagar acerca de las características que presentan los estudios más recientes sobre educación virtual, cabe resaltar la configuración de un campo sumamente complejo y heterogéneo. En este sentido, resultaría imposible reseñar aquí de modo sistemático el amplio espectro de perspectivas y enfoques, tanto teóricos como metodológicos. Una de las principales dificultades a las que nos enfrentamos actualmente, radica en que pese a que abundan los estudios empíricos, a la vez escasean enfoques multidimensionales que logren integrar esa misma heterogeneidad -de perspectivas y disciplinas- de modo consistente. De este modo, puede observarse cómo en las ciencias sociales prolifera aquella ensayística que se caracteriza por manipular un nivel teórico y conceptual fascinante, que logra sin dudas extender el horizonte de los imaginarios posibles. Un horizonte que siempre corre detrás de un mundo en permanente cambio y transformación, tanto a nivel cuantitativo como cualitativo. Mark Fisher (2018) nos advertía hace algunos años que la educación aparece como motor de la reproducción de la realidad social, en el marco de lo que él llama realismo capitalista. Así, la educación se produce en un escenario de poslexia en el que los y las adolescentes tienen la capacidad de procesar los datos cargados de imágenes del capital sin ninguna necesidad de leer (Fisher, 2018, pág. 54). Según Ficher, los docentes aparecen entonces como "facilitadores del entretenimiento y, al mismo tiempo, disciplinadores autoritarios" (2018, pág. 55). Esto muestra la clase de paradojas que enfrentemos quienes estudiamos procesos y experiencias escolares en las sociedades contemporáneas. De este modo, las dificultades y complejidades implicadas en la terea cotidiana de los cientistas sociales, no terminan de multiplicarse. Esta parece una consecuencia inevitable, toda vez que se intenta someter a escrutinio aquellas hipótesis que hoy guían gran parte de las agendas de trabajo que ponen el foco en el vínculo entre nuevas tecnologías y experiencias educativas.

Sin lugar a dudas nos enfrentamos hoy a un campo de estudios sumamente rico y heterogéneo que parece avanzar en múltiples direcciones a la vez. Sin embargo, si queremos seguir profundizando nuestro conocimiento acerca de los usos de las tecnologías y las transformaciones que están teniendo lugar en el campo educativo, se vuelve necesario reordenar ciertos términos de la discusión. A pesar del riesgo que conlleva generalizar ciertas observaciones, a modo de síntesis, considero útil reconstruir este campo de estudios en clave procesual, prestando especial atención a los cambios que se observan a nivel de los supuestos que están detrás de las investigaciones. Así, es posible identificar tres enfoques distintos, que bajo ningún aspecto deben ser considerados como mutuamente excluyentes: a) aquellos 
trabajos que abordan el uso de las nuevas tecnologías, midiendo de algún modo el impacto de estas sobre los procesos educativos; b) aquellos que dan cuenta más bien de cierta reconfiguración, lo que supone un nuevo estado de relación entre factores que se influyen recíprocamente; y c) una tercera perspectiva que toma como hipótesis de fondo la existencia de un nuevo ambiente, en el que las tecnologías aparecen ya como trasfondo necesario e insoslayable de todas las relaciones y procesos sociales.

Asumiendo este punto de vista en clave de tipos ideales, los trabajos más recientes podrían ser revisados y organizados según cuatro grandes áreas. En primer lugar, y pensando fundamentalmente en clave de impacto, podrían identificarse aquellas líneas de trabajo que se enfocan en cuestiones pedagógicas, prestando atención a la formación docente, el diseño curricular, el acompañamiento a través de tutorías virtuales (Toledo Lara, 2017) y los efectos de las nuevas tecnologías en los aprendizajes y competencias. Tomando en cuenta los distintos análisis acerca de las buenas prácticas de e-learning (Carmona Suárez \& Rodríguez Salinas, 2017) y b-learning (Valverde-Berrocoso \& Balladares Burgos, 2017), como así también de las experiencias de autorregulación y construcción de ambientes colaborativos de trabajo (Anderson, Hernández, \& Jiménez, 2018; Rodríguez Zamora \& Espinoza Núñez, 2017), sería posible identificar un segundo grupo en el que coinciden aquellos enfoques que apuntan a observar tanto el impacto como la reconfiguración de las experiencias educativas. En ese terreno híbrido podrían ubicarse también aquellos trabajos que insisten en la existencia de una brecha digital más o menos profunda, que no hace más que reconfigurar las desigualdades a escala regional y global (Escuder, 2019; Gómez Navarro, 2019; Benítez Larghi, 2020; Macchiarola, Martini, Montebelli, \& Mancini, 2018). De este modo, la inclusión/exclusión digital da cuenta de un proceso que redefine los términos en los que el Sur sufre los embates del capitalismo transnacional. Por otra parte, y apoyándose fundamentalmente en enfoques que prestan atención a la reconfiguración y a la proliferación de nuevos ambientes digitales, aparecen aquellos trabajos que analizan los entornos y plataformas virtuales de aprendizaje (Contreras-Colmenares, 2019; Toca Torres, 2019; Morado \& Ocampo Hernández, 2019; Del Prete \& Almenar, 2019), la gamificación de los procesos de aprendizaje (Melo-Solarte \& Díaz, 2018) y el uso de la realidad aumentada (Alvarez-Marin, Castillo-Vergara, Pizarro-Guerrero, \& Espinoza-Vera, 2017). Finalmente, y como parte de un enfoque más radical aún, podrían ubicarse aquellos trabajos que refieren al Big Data, siguiendo los rastros de la huella digital (Rojas-Castro, 2017). Estos trabajos se caracterizan por recurrir a aquellas herramientas de analítica que ofrecen las grandes plataformas -en lo que se conoce como Analytics o Data Mining-, lo que da cuenta de un enfoque que da por hecho la existencia de un nuevo ambiente virtual.

En el actual escenario se impone como necesario repensar el vínculo entre educación y virtualidad, habida cuenta de que las propias coordenadas en las que se venía pensando hasta ahora la educación en línea se han visto profundamente amenazadas por un contexto global de total incertidumbre, en el que influyen factores que nunca habían sido considerados hasta hoy. Es posible que estemos ante una verdadera transformación de las experiencias educativas, y por lo tanto de transformación de los términos en los que se desarrollan los procesos de socialización que, en tiempos de cuarentena, son fundamentalmente digitales. Es en este sentido que el carácter híbrido y multidimensional del campo de estudios sobre la educación virtual, puede ser aprovechado, e incluso enriquecido. Para ello se vuelve fundamental contar con datos en tiempo real que nos permitan poner a prueba las hipótesis que van guiando los procesos reflexivos.

\section{MÉTODO}

En lo que respecta al diseño metodológico, los resultados aquí presentados se desprenden de un estudio exploratorio en el que se optó por una encuesta virtual (diseñada sobre la plataforma Google Forms), de modo tal que permitiera cumplir con los criterios de viabilidad y rapidez. Esto hizo posible captar en el menor tiempo posible una muestra lo suficientemente amplia de la población bajo estudio. De este modo es que se aplicó un método de muestreo no probabilístico por bola de nieve $(n=384)$. La muestra recoge las experiencias y representaciones de docentes y estudiantes (40,9 y 59,1\%, respectivamente) de instituciones educativas de los distintos niveles educativos. Si bien aquí se reflejan los datos de docentes y estudiantes de nivel primario y secundario, son mayoritarios los de nivel terciario $(15,1 \%$ ) y universitario $(64,8 \%)$. Entre la población encuestada, el $77,1 \%$ se reconocen como mujeres, el $21,1 \%$ varones, el o,8\% como otrxs y el $1 \%$ optó por no responder acerca de su género autopercibido. Asimismo, cabe mencionar que se aseguró incluir tanto a instituciones de gestión pública $(76,8 \%)$ como privada $(23,2 \%)$, cubriendo no solo aquellas que ofrecen titulaciones bajo modalidad presencial $(93,2 \%)$ sino también semi-presencial $(4,4 \%)$ y a distancia (2,3\%). En lo que respecta a la distribución geográfica, el presente estudio abarca 15 de las 23 provincias que conforman la República Argentina, incluyendo también a la Ciudad Autónoma de Buenos Aires. Cabe resaltar en este punto, una vez más, la importancia de contar con datos que reflejen el estado de situación en un marco general caracterizado por la necesidad y la urgencia de dar respuestas frente a 
las problemáticas emergentes. En ello radica la justificación de ciertas apuestas y reparos metodológicos, que bajo condiciones diferentes, hubieran seguido otro curso.

El cuestionario, de tipo autoadministrado, fue distribuido mediante correo electrónico y aplicaciones de mensajería instantánea, lo que permitió que en pocos días se contara con resultados de alcance nacional. Las preguntas estuvieron enfocadas en las experiencias de utilización de plataformas y aplicaciones, en el marco de los procesos de enseñanza y aprendizajes (antes y durante la implementación de políticas de prevención y distanciamiento social); la evaluación de los conocimientos acerca de estas plataformas y las propuestas formativas de cada institución; tipos de dispositivos que utilizan habitualmente; y dificultades o problemas a los que se han enfrentado durante la implementación de modalidades de enseñanza virtual. En cuanto al procesamiento y análisis de los datos recogidos, cabe mencionar finalmente que se recurrió a software estadístico específico de distribución libre bajo Licencia Pública General de GNU.

\section{RESULTADOS}

Al prestar atención a la utilización de las distintas clases de aplicaciones y plataformas que se utilizan habitualmente en el marco de los procesos de enseñanza y aprendizaje, se han identificado cuatro grandes grupos. En primer lugar, están aquellos de uso amplio o general (Tipo 1) como WhatsApp, YouTube y Drive. En segundo lugar, las plataformas o entornos virtuales de uso educativo específico, como Moodle y Classroom (Tipo 2). En tercer lugar, las aplicaciones de videollamadas o videoconferencias como Zoom, Meet y Jitsi, entre otras (Tipo 3). Finalmente, aquellas plataformas o sitios que funcionan como repositorios oficiales de contenidos educativos (Tipo 4), que en el caso de Argentina pueden ser ejemplificados a partir de los sitios educ.ar y encuentro.gob.ar, ambos nacidos del seno del Ministerio de Educación de la Nación. Sin dudas esta clasificación no es excluyente, y responde a un escenario y contexto particular que debe ser revisado y tenido en cuenta especialmente. Con la excepción de WhatsApp, en los cuatro tipos de aplicaciones y plataformas se imponen los docentes como aquellos que las utilizaban con mayor frecuencia antes de la situación de cuarentena obligatoria. Una diferencia mínima en los casos de YouTube y Classroom, algo mayor en Drive y Moodle y más pronunciada aún en relación al uso de aplicaciones y plataformas Tipo 3 y 4 (ver Gráfico 1).

Gráfico 1. Utilización de aplicaciones o plataformas para educación en línea, antes de la cuarentena.

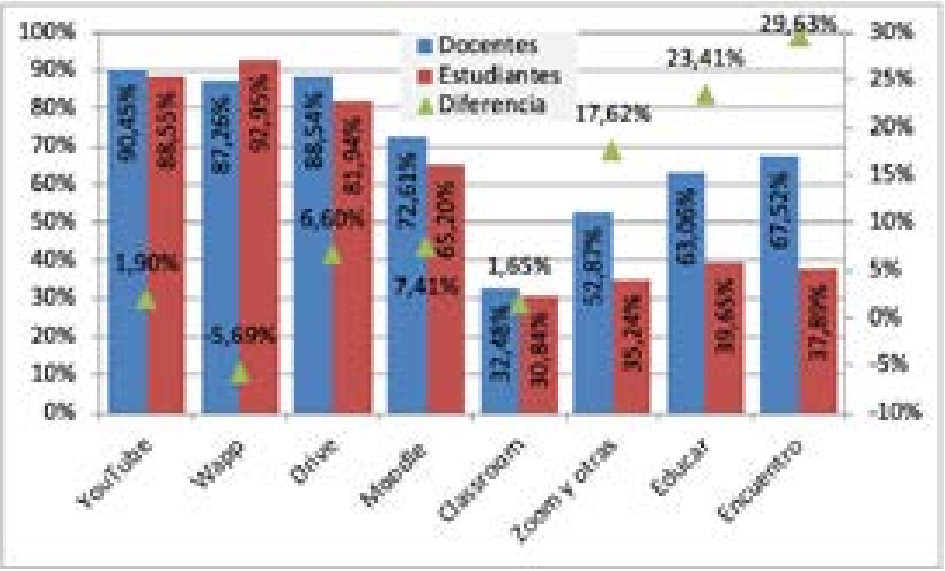

Fuente: elaboración propia.

Por otra parte, al indagar acerca de los conocimientos que poseen los docentes y estudiantes acerca de las aplicaciones y plataformas aplicadas a procesos educativos, cabe destacar una diferencia mínima aunque relevante. En una escala de 1 a 10, siempre medida desde la percepción de los propios actores, puede observarse una mejor estimación por parte de los docentes. Como puede apreciarse en el Gráfico 2, estos últimos se imponen en las puntuaciones más altas. El 58,6\% de los docentes puntúan sus conocimientos entre 7 y 10 , frente al $51,55 \%$ de los estudiantes. 
Gráfico 2. Conocimientos sobre aplicaciones y plataformas

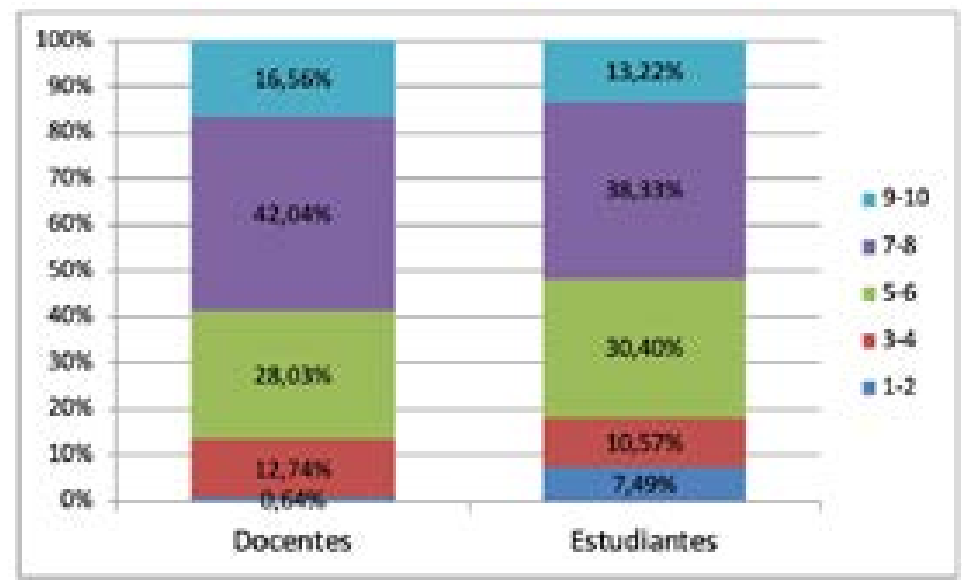

Fuente: elaboración propia.

Al prestar atención a la utilización de aplicaciones o plataformas con fines educativos durante la primera fase de la cuarentena, nuevamente se imponen los docentes, a excepción de WhatsApp y Moodle. Al momento de aplicarse el cuestionario más del $72 \%$ de los docentes habían utilizado alguna de las herramientas de Tipo 1, mientras que el 69,05\% habían utilizado alguna de las herramientas de Tipo 2 (Moodle y Classroom). Asimismo, en el gráfico 3 podemos observar que el $54,7 \%$ de los docentes y el 39,6\% de los estudiantes ya habían utilizado alguna de las herramientas Tipo 3. La diferencia en favor de los docentes se explica en parte por la realización de reuniones organizativas previo al inicio de clases, que en algunas facultades y años de cursada tendría lugar recién a partir del 13 de abril.

Gráfico 3. Utilización de aplicaciones o plataformas para educación en línea, durante la primera fase de cuarentena.

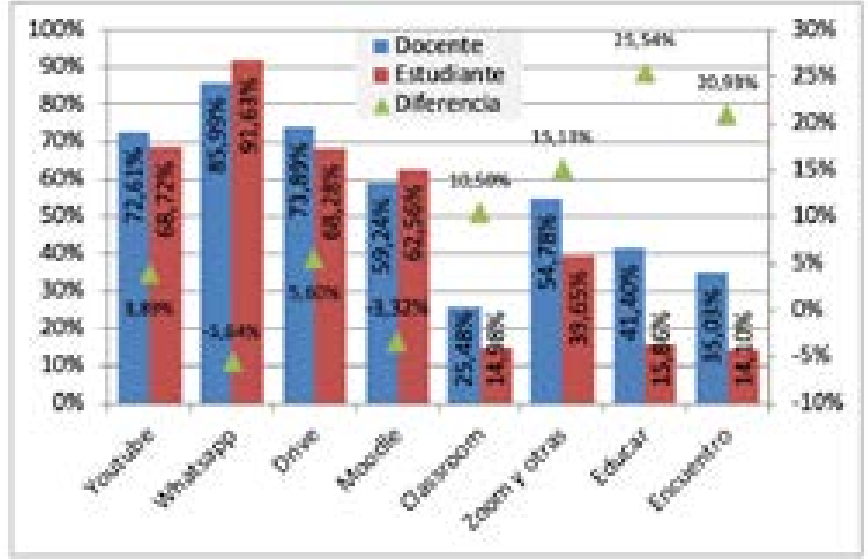

Fuente: elaboración propia.

Al detenernos en la evaluación que hacen docentes y estudiantes respecto de la propuesta formativa mediada por las nuevas tecnologías, se resalta una buena recepción en general. Cabe destacar que los docentes que la valoran como Muy Buena, representan el 19,11\% frente al 8,3\% de los estudiantes. Al otro extremo del gráfico 4 podemos observar que el $21,6 \%$ de los docentes y el $24,2 \%$ de los estudiantes califican la propuesta de sus instituciones como Mala o Muy Mala, siendo más severos los docentes en cuanto a este último calificativo. 
Gráfico 4. Evaluación de la propuesta formativa mediada por las nuevas tecnologías de la institución implementó hasta ahora en el marco de la cuarentena

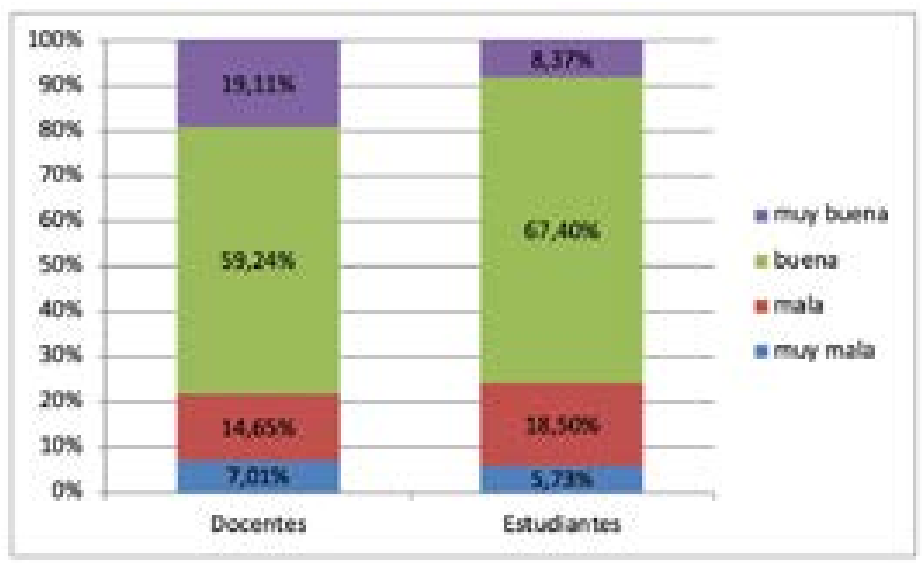

Fuente: elaboración propia.

Siguiendo con las apreciaciones y evaluaciones de los actores sobre sus experiencias, pensadas en clave institucional, podemos observar que el nivel de satisfacción respecto al acompañamiento institucional es mayor entre los docentes, alcanzando el $43,79 \%$ frente al $25 \%$ de los estudiantes. Sin embargo, la mirada más crítica sobre las instituciones en las que desarrollan su actividad también se impone con mayoría relativa entre los docentes, de los cuales el $17 \%$ considera que es Insuficiente, frente al $12 \%$ de los y las estudiantes.

Tabla 1. Acompañamiento institucional en la utilización de recursos y plataformas

\begin{tabular}{|l|c|c|c|c|c|c|c|}
\hline & \multicolumn{2}{|c|}{$\begin{array}{l}\text { No, fue completa- } \\
\text { mente insuficien- } \\
\text { te }\end{array}$} & \multicolumn{2}{|c|}{$\begin{array}{c}\text { Sí, aunque podría } \\
\text { mejorarse }\end{array}$} & \multicolumn{2}{c|}{$\begin{array}{c}\text { Si, conté con el } \\
\text { acompañamiento } \\
\text { necesario }\end{array}$} & \\
\cline { 2 - 8 } & $\mathrm{N}$ & $\%$ & $\mathrm{~N}$ & $\%$ & $\mathrm{~N}$ & $\%$ & Total \\
\hline Docentes & 26 & 17,0 & 60 & 39,2 & 67 & 43,8 & 153 \\
\hline Estudiantes & 24 & 12,0 & 126 & 63,0 & 50 & 25,0 & 200 \\
\hline
\end{tabular}

Fuente: elaboración propia.

Al detenernos en la cuestión de los dispositivos que utilizan, hay varios datos que deben ser tenidos en cuenta. Por un lado, recurrir a una PC o Laptop prestada es algo poco frecuente entre los docentes, triplicándose este indicador para el caso de los estudiantes. Resalta, por otro lado, el hecho de que el $74,8 \%$ de los docentes tengan acceso a una PC o Laptop de uso personal, frente al $54 \%$ de los estudiantes. Finalmente, es necesario insistir una vez más en la importancia que siguen teniendo los Smartphone como principal herramienta de acceso a Internet. Como podemos observar en el gráfico 5, casi la tercera parte de los estudiantes utilizan estos dispositivos para acceder a las plataformas que median en procesos de enseñanza y aprendizaje, mientras solo el 10\% de los docentes lo hacen.

Gráfico 5. Dispositivo utilizado habitualmente en los procesos de enseñanza y aprendizaje

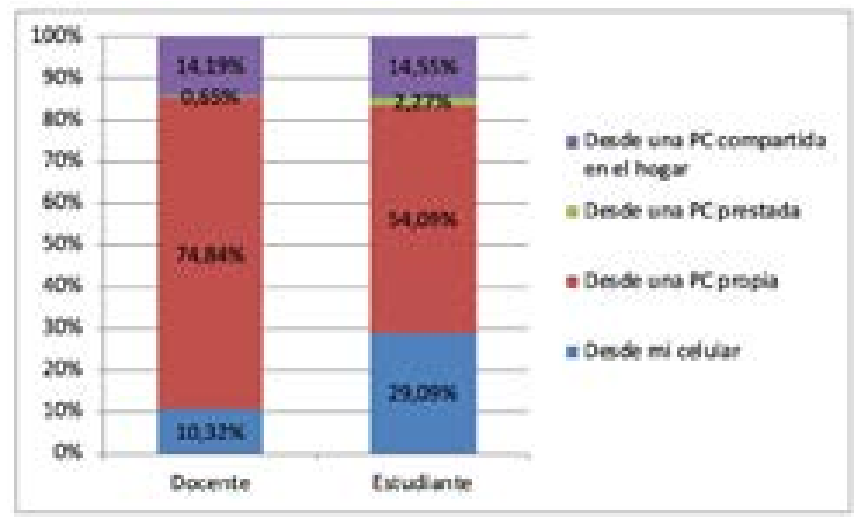

Fuente: elaboración propia. 
Finalmente, al prestar atención a las dificultades a las que se enfrentan habitualmente docentes y estudiantes, es posible identificar un cuadro de situación ciertamente desigual. A través del gráfico 6 se intenta ilustrar los distintos modos en que se ven afectados los actores según la clase de problemas que influyen en sus experiencias de enseñanza y aprendizaje virtual. De este modo, los estudiantes sufren en mayor medida problemas vinculados a la carga o descarga de datos (por saturación, caída del sistema, demoras, problemas con el servidor, etc.), cuestiones de software (autorizaciones, actualizaciones, derechos, virus, etc.), hardware y de organización institucional. Los docentes, por su parte sufren en mayor medida las consecuencias de la falta de equipamientos adecuados en sus respectivas instituciones. virtual.

Gráfico 6. Principales dificultades que afectan a estudiantes y docentes, vinculadas a la educación

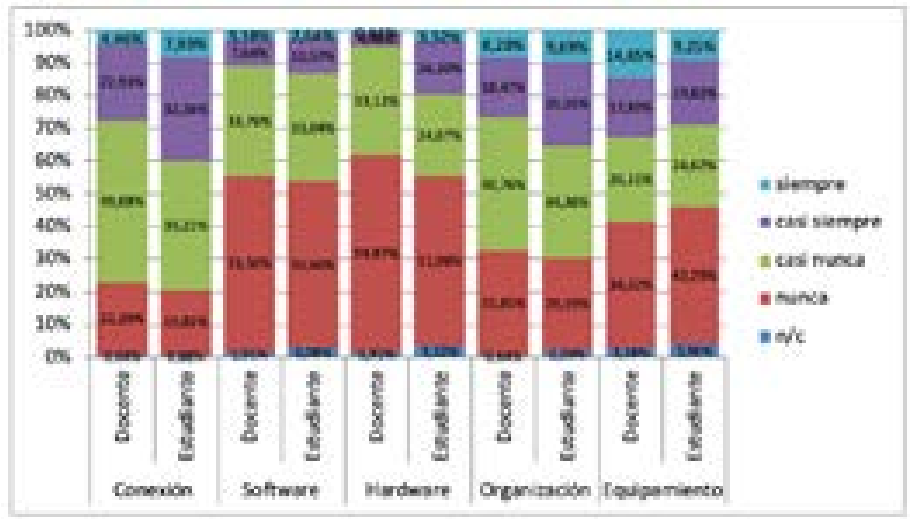

Fuente: elaboración propia.

\section{CONCLUSIONES}

Los datos que hemos presentado hasta aquí permiten hacernos una idea del estado de situación actual como si se tratara de una fotografía, en medio de un proceso sin dudas abierto. Las medidas de prevención y aislamiento físico aplicadas en nuestra región, como es el caso la cuarentena iniciada en Argentina el 20 de marzo, han influido directa e intempestivamente en los modos en que las instituciones educativas debieron afrontar el calendario académico de 2020. Sin diagnósticos previos, los principales actores decisores se encontraron ante la necesidad de reconducir los procesos de enseñanza y aprendizaje hacia entornos y plataformas virtuales. De este modo, en medio del cierre de escuelas y universidades se abrieron miles de aulas virtuales. Aún desconociendo la dimensión de la brecha digital en cada contexto, docentes, estudiantes, padres, directivos y funcionarios emprendieron un sinuoso camino casi a oscuras.

Resta evaluar aún los efectos inmediatos, como así también el impacto a mediano y largo plazo, de las nuevas experiencias que tuvieron lugar en medio de la pandemia global del virus Covid-19. Los datos que hemos presentado aquí sugieren que al menos durante la primera fase de la cuarentena, los estudiantes debieron enfrentar una situación de relativa precariedad y desigualdad, lo que supone la imposibilidad de una incorporación plena a las experiencias virtuales de aprendizaje. Pese a lo compulsivo que resultó este proceso de digitalización de las trayectorias educativas, las experiencias de uso previas, como así también los conocimientos acerca del funcionamiento de las aplicaciones y plataformas educativas, demostraron una marcada diferencia entre los actores, en perjuicio de los propios estudiantes. La mirada acerca de la propuesta formativa y el acompañamiento de cada institución, confirma que los estudiantes se encontraron desprotegidos y sin las herramientas adecuadas para enfrentar una situación tan novedosa como esta. Finalmente, las desiguales condiciones de acceso a los dispositivos adecuados para afrontar las nuevas experiencias educativas virtuales, y el impacto diferencial de las dificultades técnicas que habitualmente afectan los modos de habitar y navegar por la web, no hicieron más que confirmar y refrescar un escenario profundamente inclinado y, por lo tanto, con mayores problemas de conexión para unos que para otros.

\section{Conflict of interest}

No potential conflict of interest is reported by the author(s).

\section{Funding}

There is no financial assistance in studies from external parties.

\section{Acknowledgements}

Proyecto de Investigación: "Trayectorias de Nivel Superior" de la Universidad Nacional de Villa María. 


\section{REFERENCIAS}

Alvarez-Marin, A., Castillo-Vergara, M., Pizarro-Guerrero, J., et al. (2017). Realidad Aumentada como Apoyo a la Formación de Ingenieros Industriales. Formación Universitaria, 10(2), 31-42. http://dx.doi. org/10.4067/So718-50062017000200005

Anderson, M., Hernández, I., y Jiménez, M. (2018). Aulas Digitales en la Educación Superior: Caso México. Formación Universitaria, 11(5), 93-104. http://dx.doi.org/10.4067/S0718-50062018000500093

Benítez Larghi, S. (2020). Desafíos de la inclusión digital en Argentina. Revista de Ciencias Sociales, 33(46),131154. https://doi.org/10.26489/rvs.v33i46.7

Carmona Suárez, E. J., y Rodríguez Salinas, E. (2017). Buenas prácticas en la educación superior virtual a partir de especificaciones de estándares de e-Learning. Sophia, 13(1), 13-26. http://dx.doi. org/10.18634/sophiaj.13v.1i.345

Contreras-Colmenares, A. F. D. (2019). Ambientes Virtuales de Aprendizaje: dificultades de uso en los estudiantes de cuarto grado de primaria. Prospectiva. Revista de Trabajo Social e intervención social, (27), 215-240. https://doi.org/10.25100/prts.voi27.7273

Del Prete, A., y Almenar, J. C. (2019). Las plataformas de formación virtual: algunas variables que determinan su utilización. Apertura, 11(2), 138-153. http://doi.org/10.32870/Ap.v11n2.1521

Escuder, S. (2019). Regionalización de la brecha digital. Desarrollo de la infraestructura de las TIC en Latinoamérica y Uruguay. Paakat: Revista de Tecnología y Sociedad, 17(9), 1-23. http://dx.doi. org/10.32870/pk.a9n17.356

Fisher, M. (2018). Realismo capitalista: ¿No hay alternativa? Buenos Aires: Caja Negra.

Gómez Navarro, D. A. (2019). Uso de las tecnologías de la información y la comunicación por universitarios mayas en un contexto de brecha digital en México. Región y sociedad. (31)e1130, 1-25 http://dx.doi. org/10.22198/rys2019/31/1130

Hernández, R., Sánchez, I., Zarate, J., et al. (2019). Tecnología de Información y Comunicación (TIC) y su práctica en la evaluación educativa. Propósitos y Representaciones, 7(2), 1-10. http://dx.doi. org/10.20511/pyr2019.v7n2.328

Macchiarola, V., Martini, C., Montebelli, A. E., y Mancini, A. A. (2018). Inclusión digital educativa en escuelas secundarias argentinas. Un estudio evaluativo. Ciencia, Docencia y Tecnología, 29(57), 149-175. http:// www.pcient.uner.edu.ar/cdyt/article/view/335

Melo-Solarte, D.,y Díaz, P. (2018). El AprendizajeAfectivoy la Gamificación en Escenarios de Educación Virtual. Información tecnológica, 229(3), 37-248. http://dx.doi.org/10.4067/S0718-07642018000300237

Morado, M. F., y Ocampo Hernández, S. (2019). Una experiencia de acompañamiento tecno-pedagógico para la construcción de Entornos Virtuales de Aprendizaje en Educación Superior. Educación. 43(1), http://dx.doi.org/10.15517/revedu.v43i1.28457

Rodríguez Zamora, R., y Espinoza Núñez, L. A. (2017). Trabajo colaborativo y estrategias de aprendizaje en entornos virtuales en jóvenes universitarios . Revista Iberoamericana para la Investigación y el Desarrollo Educativo, 7(14), 86-109. http://dx.doi.org/10.23913/ride.v7i14.274

Rojas-Castro, P. (2017). Learning analytics: una revisión de la literatura. Educación y Educadores, 20(1), 106128. http://dx.doi.org/10.5294/edu.2017.20.1.6

Toca Torres, C. E. (2019). Los entornos de aprendizaje inmersivo y la enseñanza a ciber-generaciones. Educação e Pesquisa, 45(19), 1-20. https://doi.org/10.159o/s1678-4634201945187369

Toledo Lara, G. (2017). La virtualidad en la tutoría docente: Una aproximación a su análisis desde la universidad española. Revista Digital de Investigación en Docencia Universitaria, 11(2), 323-342. http:// dx.doi.org/10.19083/ridu.11.509

Valverde-Berrocoso, J., y Balladares Burgos, J. (2017). Enfoque sociológico del uso del b-learning en la educación digital del docente universitario. Sophia, (23), 123-140. http://dx.doi.org/10.17163/soph. n23.2017.04

\footnotetext{
Author:

Andrés Hernández. Becario Postdoctoral del Consejo Nacional de Investigaciones Científicas y Técnicas, Instituto Académico Pedagógico de Ciencias Sociales, Universidad Nacional de Villa María, Córdoba, Argentina
} 